\title{
TTR
}

Traduction, terminologie, re?daction

\section{Traduction de The Three Companions de W. H. Auden}

\section{Christine Klein-Lataud}

Volume 12, numéro 2, 2e semestre 1999

Poésie, cognition, traduction II - Autour d'un poème de W. H. Auden

Poetry, Cognition, Translation II — On a Poem by W. H. Auden

URI : https://id.erudit.org/iderudit/037373ar

DOI : https://doi.org/10.7202/037373ar

Aller au sommaire du numéro

\section{Éditeur(s)}

Association canadienne de traductologie

\section{ISSN}

0835-8443 (imprimé)

1708-2188 (numérique)

Découvrir la revue

Citer cet article

Klein-Lataud, C. (1999). Traduction de The Three Companions de W. H. Auden. TTR, 12(2), 77-81. https://doi.org/10.7202/037373ar d'utilisation que vous pouvez consulter en ligne.

https://apropos.erudit.org/fr/usagers/politique-dutilisation/ 


\section{Traduction de The Three Companions de W. H. Auden}

\section{Christine Klein-Lataud}

Ayant accepté avec enthousiasme de participer à l'atelier de traduction poétique organisé par Annie Brisset, j'attendais avec impatience le poème qui en serait l'objet. J'avoue m'être un peu inquiétée en recevant "The Three Companions", condensé de difficultés de tous ordres, poème que je trouvais fascinant mais dont le charme venait en partie de son opacité même, de son mystère.

Les quelques lignes qui suivent n'ont d'autre prétention que de restituer la démarche qui a abouti à ma traduction.

En un premier temps, comme je le fais toujours, je me suis laissée aller au texte, attentive seulement aux émotions et aux échos qu'il suscitait en moi. Son côté sombre, le paysage mortifère qu'il campe évoquaient les ballades germaniques, le Roi des Aulnes, la rencontre du Chevalier et de la Mort, comme dans la gravure de Dürer. L'oiseau dans l'arbre tourmenté et les taches atroces sur la peau suscitaient l'horreur comme le Corbeau et le Masque de la Mort Rouge d'Edgar Poe. Plus obscurément et idiosyncratiquement, me revenaient des chansons d'enfant terrifiantes, en particulier la complainte du Grand Lustucru (qui repasse et passera...), des souvenirs de peurs, d'ombres et de bruits dans la nuit, de la mort qui rôde. Tout cela a pour moi déterminé le ton, la couleur de ma future traduction. La leçon optimiste du texte (le refus du sujet de se laisser intimider dans sa quête du monde) ne m'est apparue qu'en un second temps, au terme d'une étude éclairée par des textes critiques : pour moi, les tonalités sombres de la terreur restent dominantes. 
En un deuxième temps, je me suis documentée sur le texte.' J'ai lu The Orators, le recueil disparate dont ce poème constitue la conclusion. Cette démarche rationnelle ne m'a pas été très utile, en fait, parce que le lien avec l'ensemble est loin d'être évident et que W. $\mathrm{H}$. Auden a par la suite republié ce poème dans tous ses recueils poétiques, l'isolant ainsi de son contexte initial et lui donnant valeur autonome.

La consultation de ces recueils de poèmes ainsi que de quelques études critiques m'a révélé la prédilection d'Auden pour la ballade et, d'une façon générale, son goût pour la poésie populaire. Cela m'a confirmée dans ma première décision importante : ne pas traduire en vers libres mais adopter une forme régulière en français, pour conserver l'aspect de ballade populaire du texte anglais. En l'absence d'une forme fixe correspondante, j'ai choisi l'alexandrin. Ma position est diamétralement opposée à celle d'Annie Brisset, qui exclut radicalement l'alexandrin des formes de traduction possibles, l'accusant de « permettre un phagocytage du poème par l'aura littéraire et culturelle française $)^{2}$. C'est précisément parce que c'est le mètre français le plus habituel, celui que tous gardent dans l'oreille grâce à l'école que je l'ai choisi, désirant recréer un effet de familiarité chez le lecteur français, tout comme Auden l'a fait pour le lecteur anglais. Le sentiment $d$ ' "inquiétante étrangeté " (pour reprendre un terme de Freud) est intensifié par le contraste entre la familiarité de la forme et le caractère terrifiant du contenu.

Ayant donc adopté l'alexandrin (et je suis frappée par le fait que les deux autres traductrices aient fait le même choix), j'ai voulu également respecter la coupe classique à l'hémistiche ${ }^{3}$. Le balancement de séquences isorythmiques me paraissait refléter à sa manière les pentamètres anglais et contribuer par sa répétition au côté hallucinatoire du poème.

' Pour l'analyse textuelle du poème, je me suis appuyée sur deux articles d'Annie Brisset, "Poésie : le sens en effet. Étude d'un translème ", Meta, vol. 29, $\mathrm{n}^{\circ}$ 3, septembre 1984, pp. 259-266, et "Intertextualité et traduction. Un poème de W. H. Auden », Le Journal Canadien de Recherche Sémiotique, vol. VIII, $\mathrm{n}^{\text {os }} 1 \& 2$, pp. 201-214.

${ }^{2}$ Annie Brisset, « Intertextualité... », p. 213.

${ }^{3}$ Une exception, le $3^{\mathrm{e}}$ vers de la $3^{\mathrm{e}}$ strophe, que j'ai dû me résigner à couper 43-5. 
Par contre, j'ai renoncé à la rime, parce que son utilisation m'entraînait dans toutes sortes de transformations que j'estimais abusives.

Le deuxième aspect essentiel du poème que j'ai voulu rendre en priorité, c'est sa force allitérative. Elle est, bien sûr, particulièrement évidente dans la série des couples antagonistes reader/rider, fearer/farer, horror/hearer, sur laquelle je reviendrai plus bas. Mais elle préside à tout le poème. Paronomases comme midden/madden, path/pass, looking/lacking; allitérations comme fatal/furnaces, gap/grave, granite/grass, etc. Après ma première ébauche, j'ai donc systématiquement retravaillé ma traduction pour l'enrichir en allitérations chaque fois que je le pouvais, en cherchant des synonymes ou parasynonymes. J'en donnerai deux exemples. Pour le vers 4 de la $1^{\text {ère }}$ strophe, j'avais d'abord écrit: "Cette faille est la tombe où retournent les grands " et $j$ 'ai remplacé " tombe " par " fosse " pour l'allitération avec « faille ». Le vers 3 de la $3^{e}$ strophe : "Derrière toi furtive s'est glissée une ombre " est devenu "Derrière toi sournoise s'est glissée une ombre ", pour l'allitération avec "glissée » et l'assonance avec "toi ${ }^{4}$. Bref, j'ai voulu un texte aussi riche en allitérations que possible, même si, bien sûr, celles-ci ne portent pas forcément sur les mêmes mots que le texte anglais (ex. : au vers 4 de la $3^{\mathrm{e}}$ strophe, au lieu de l'allitération spot/skin, j'ai « peau »/« peste ».

Pour les couples d'acteurs reader/rider, fearer/farer, horror/hearer, il fallait essayer de trouver des couples de lexèmes aussi paronomastiques que possible, ayant tous le même nombre de syllabes et dotés d'une valeur sémantique contradictoire. La régularité du paradigme des trois couples devait être respectée à travers toutes les strophes. C'est ainsi que j'ai renoncé au beau couple "Escolier »/«Écuyer» parce que seuls les mots dissyllabiques offraient assez de choix pour les couples des strophes suivantes. Outre celles de la forme, toutes sortes de contraintes pesaient sur le choix des lexèmes : la lisibilité contemporaine, le registre et les connotations. Ainsi pour traduire Rider, "Viateur" aurait été parfait, puisqu'il signifie étymologiquement voyageur, mais il n'est plus compréhensible. 'Quant à "Coureur », le mot maintenant évoque plus les stades ou l'amateur de jupons que celui qui court le monde ou le

\footnotetext{
${ }^{4}$ Bénéfice secondaire : d'un point de vue sémantique, « sournois 》 a le sème péjoratif que ne contient pas " furtif ». La recherche sur le signifiant peut ainsi déboucher sur une amélioration du signifié.
} 
bois. Faute de mieux, j'avais arrêté mon choix sur " Rôdeur ", qui implique le mouvement mais qui a le grave inconvénient d'être péjoratif. La solution m'est venue lors de l'atelier et j'ai emprunté "Marcheur " à une des participantes. Pour le couple Fearer/Farer, j'aurais aimé, opposé à " Froussard ", " Routard ", celui qui court les routes, mais outre ses connotations péjoratives, "Routard" fait beaucoup trop contemporain. Je me suis résignée à traduire Farer par "Curieux » en extrayant une des notions associées à celle de voyageur, et pour faire pendant à " Peureux » par l'assonance finale. Après avoir hésité entre les couples "Craintif»/«Curieux» et "Peureux »/« Curieux ", je me suis décidée pour la deuxième solution qui m'a semblé phonétiquement plus riche.

Pour tous ces acteurs, j'ai gardé la majuscule à l'initiale qui en fait des personnages allégoriques, retrouvant ainsi une tradition poétique ancienne qui existe aussi bien dans la littérature française qu'anglaise. Je pense en particulier au Roman de la Rose, à la poésie des Grands Rhétoriqueurs, mais aussi à Ronsard (" Mais ils ont beau courir, car Vieillesse les suit ») ou à Victor Hugo ("Le spectre Rien levait sa tête hors du gouffre "). Le procédé allégorique donne une existence individuelle et, partant, plus de vie aux acteurs génériques; il $\mathrm{m}$ 'a en outre permis, en l'absence de nom d'agent satisfaisant ("Écouteur ", par exemple, a trois syllabes) de traduire Hearer par "l'Oreille" (en opposition avec "l'Horreur»). La trouvaille de Charlotte Melançon, "Veilleur ", me plaît, et je serais prête à adopter le couple " Horreur »/« Veilleur », mais je maintiens ma solution pour le plaisir de la diversité...

Au total, donc, je n'ai modifié qu'un mot au terme de l'atelier. Il ne s'agit pas d'obstination à défendre mes solutions mais de cohérence. Ayant choisi l'alexandrin coupé à l'hémistiche, je disposais de peu de souplesse pour des remplacements. En outre, les principes directeurs exposés ci-dessus (en particulier la recherche de la paronomase et des allitérations en général) m'avaient amenée à tisser le texte le plus serré possible : il n'y avait donc plus guère de « jeu ».

J'emprunterai ma conclusion à Annie Brisset: " [L]e texte poétique ne peut être idéalement restitué comme totalité signifiante que par la "somme de ses traductions significativement différentes" " 5 .

\footnotetext{
${ }^{5}$ Annie Brisset, « Poésie : le sens en effet... », p. 266.
} 
À chaque lectrice et lecteur de juger et surtout de jouer à son tour, car si la critique est facile...

Collège universitaire Glendon

\section{LES TROIS COMPAGNONS}

Où vas-tu donc ainsi? dit Liseur à Marcheur, Fatale est la vallée quand flambent les fabriques, Là-bas est le fumier à l'odeur infâmante

Cette faille est la fosse où retournent les grands.

T'imagines-tu donc, dit Peureux à Curieux Que le soir attendra que tu sois à la passe Que ton cil diligent discernera le vide Que ton pas glissera du granit au gazon?

Qu'était donc cet oiseau? dit l'Horreur à l'Oreille As-tu vu cette forme en ces arbres tordus? Derrière toi sournoise s'est glissée une ombre. Ces taches sur ta peau sont une peste atroce.

Hors de cette maison, dit Marcheur à Liseur Jamais tu ne pourras, dit Curieux à Peureux. C'est toi qu'ils traquent ici, dit l'Oreille à l'Horreur, Et il les laissa là, et il les laissa là.

Christine Klein-Lataud : York University, Glendon College, École de traduction, 2275, Bayview Avenue, Toronto (Ontario) M4N 3M6 Courriel : klataud@venus.yorku.ca 